\title{
Modeling and Analysis of Wireless Electro-mechanical Energy Transfer and Conversion Using Resonant Inductive Coupling
}

\author{
Yasutaka Fujimoto \\ Department of Electrical and Computer Engineering \\ Yokohama National University \\ Yokohama 240-8501 Japan \\ Email: fujimoto@ynu.ac.jp
}

\begin{abstract}
This paper presents a basic model of an electromechanical energy conversion system using the resonant inductive coupling as well as the power transfer system between the coils. The proposed system utilizes two sets of two-phase air-core coils both for the stator and the mover. There exists parasitic capacitance of the coils which is connected in series. Thus the whole system has certain resonant frequencies. Two-phase voltage source connected to the primary coils is driven at the resonant frequency. Unlike conventional wireless power transfer systems using resonant inductive coupling, the proposed system can generate moving flux field. The proposed system is similar to the induction machine but it is driven at very high resonant frequency and the slip ratio is also quite high. Tendency of the generated force of the mover obtained by the FEA simulation shows that can be explained by the model derived.
\end{abstract}

\section{INTRODUCTION}

In recent years, wireless power transfer technology using resonant inductive coupling [1]-[14] has attracted attention. This technology, compared with the conventional wireless power transmission technology using the magnetic induction [15]-[19], the transmission distance is relatively long about $1 \mathrm{~m}$. The system consists of two coils at the primary and secondary side of magnetically coupled weakly, and a capacitor connected to the coils. In some cases, it connects actual capacitors in addition to the parasitic capacitance of the coils. It is found to be able to explain the frequency and efficiency of power which can be transmitted precisely by equivalent concentrated circuit. There exists the resonant frequencies by which the impedance from the primary side becomes minimal. If the $\mathrm{Q}$ value is high enough, large power can be transmitted at the resonant frequency.

The idea in this paper is to apply the resonant inductive coupling to the electro-mechanical energy conversion system as well as the power transfer system between the coils. Based on the best knowledge of the authors, studies utilizing magnetic resonant coupling dealing with both power transfer and electromechanical energy conversion in a unified manner are not found so far. In our proposed system, totally four coils are utilized. Two primary coils at the stator are allocated as shown in Fig. 1. The phase of two coils are spatially shifted 90degree. Two secondary coils at the mover are allocated as well as the primary side. The primary coils are driven by two sinusoidal voltage sources whose phase difference is 90 degree, which generate moving flux field. The frequency of the

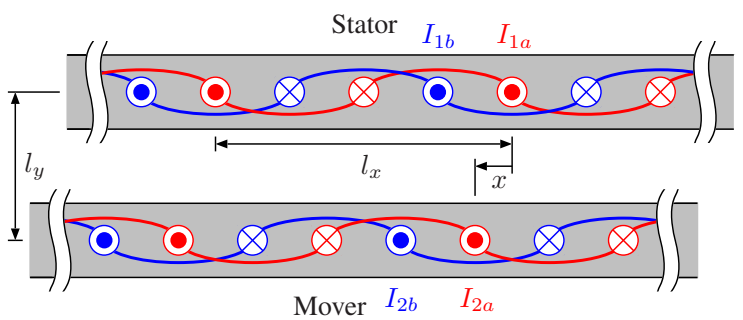

Fig. 1. A basic model of the electric machine.

voltage sources is near the resonant frequency of the system. When connecting the output end on the secondary side, it behaves as the induction machine. However, compared to the conventional induction machines, the slip ratio of the proposed machine is quite high which is near $100 \%$. This is because the frequency of the voltage sources is near the resonant frequency, which is generally between several hundreds $\mathrm{kHz}$ and ten and several $\mathrm{MHz}$, and the frequency of the mover is typically less than $10 \mathrm{kHz}$. Differences between the proposed machine and conventional induction machines are summarized as follows.

- Capacitors exist in the primary side and the secondary side, and the circuit is driven at a high frequency near the resonant frequency.

- Since the speed of the secondary side mover with respect to the driving frequency is quite small, a slip is extremely big.

- It is possible to have a large gap between the primary side and the secondary side.

- When the primary side and the secondary side are aircore coil, generated force is small.

In this paper, a basic model of the electric machine with resonant inductive coupling is proposed and generation of force is confirmed by FEA simulations.

The rest of this paper is organized as follows. Section II presents a mathematical model of the proposed electric machine where voltage equation and force equation are derived. Section III describes resonant frequency of the whole circuit considering the effect of the speed of the secondary side mover. Section IV demonstrates simulation results by using Finite 


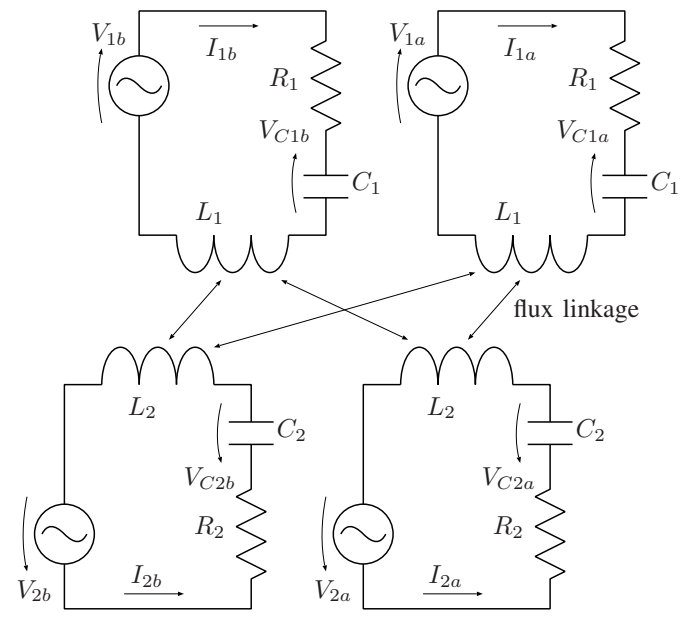

Fig. 2. The equivalent circuit of the electric machine.

Element Analysis (FEA) and generation of force is confirmed, and Section $\mathrm{V}$ concludes the paper.

\section{MODEL}

Consider four coils located as shown in Fig. 1. Twophase primary coils are fixed on the stator and the other twophase secondary coils are mounted on the mover, where $x$ is displacement of the mover, $l_{x}$ is the pitch length corresponding to $2 \pi$ radian in electric angle of the primary two-phase coils, and $l_{y}$ is the gap length between the primary coils and the secondary coils. Air-core coils are assumed in this paper. If we utilize the open-end coils both in the primary side and the secondary side, there exists parasitic capacitance of the coils which is connected in series. The equivalent circuit of this model as shown in Fig. 2. The inductive coupling between the primary coils and the secondary coils are variable according to the displacement of the mover. The inductance matrix of the coils in Fig. 2 can be described as follows.

$$
\boldsymbol{L}=\left[\begin{array}{cccc}
L_{1} & 0 & M_{0} \cos \theta & -M_{0} \sin \theta \\
0 & L_{1} & M_{0} \sin \theta & M_{0} \cos \theta \\
M_{0} \cos \theta & M_{0} \sin \theta & L_{2} & 0 \\
-M_{0} \sin \theta & M_{0} \cos \theta & 0 & L_{2}
\end{array}\right]
$$

where $L_{1}$ and $L_{2}$ are self-inductances of the primary coils and the secondary coils, respectively. $M_{0}$ is the maximum value of a mutual inductance between the primary coils and the secondary coils. $\theta=2 \pi x / l_{x}$ is the electric angle of the mover. The self-inductances and the mutual inductance are given from the magnetic field distribution. Details can be found in the Appendix.

The voltage equation of the equivalent circuit can be described as follows.

$$
\begin{aligned}
\boldsymbol{V} & =\boldsymbol{R} \boldsymbol{I}+\frac{d}{d t}(\boldsymbol{L} \boldsymbol{I})+\boldsymbol{V}_{C} \\
\boldsymbol{I}_{C} & =\boldsymbol{C} \frac{d \boldsymbol{V}_{C}}{d t}
\end{aligned}
$$

where

$$
\begin{aligned}
\boldsymbol{V} & =\left[\begin{array}{llll}
V_{1 a} & V_{1 b} & V_{2 a} & V_{2 b}
\end{array}\right]^{T} \\
\boldsymbol{I} & =\left[\begin{array}{llll}
I_{1 a} & I_{1 b} & I_{2 a} & I_{2 b}
\end{array}\right]^{T} \\
\boldsymbol{R} & =\operatorname{diag}\left(R_{1}, R_{1}, R_{2}, R_{2}\right) \\
\boldsymbol{C} & =\operatorname{diag}\left(C_{1}, C_{1}, C_{2}, C_{2}\right) \\
\boldsymbol{V}_{C} & =\left[\begin{array}{llll}
V_{C 1 a} & V_{C 1 b} & V_{C 2 a} & V_{C 2 b}
\end{array}\right]^{T}
\end{aligned}
$$

and $V_{1 a}, V_{2 a}, V_{1 b}$, and $V_{2 b}$ are terminal voltages of the coils, $I_{1 a}, I_{2 a}, I_{1 b}$, and $I_{2 b}$ are currents flowing each circuits, $R_{1}$ and $R_{2}$ are resistance of the primary coils and the secondary coils, $C_{1}$ and $C_{2}$ are parasitic capacitances of the primary coils and the secondary coils, and $V_{C 1 a}, V_{C 2 a}, V_{C 1 b}$, and $V_{C 2 b}$ are voltages of those capacitors.

Generated force along with direction of the displacement $x$ is described as follows.

$$
F=\frac{1}{2} \boldsymbol{I}^{T} \frac{\partial \boldsymbol{L}}{\partial x} \boldsymbol{I}=\frac{d \theta}{d x} \frac{1}{2} \boldsymbol{I}^{T} \frac{\partial \boldsymbol{L}}{\partial \theta} \boldsymbol{I} .
$$

Assume that the currents flowing through the coils are sinusoidal and given by

$$
\begin{aligned}
I_{1 a} & =I_{1} \sin \left(\omega_{1} t\right) \\
I_{1 b} & =I_{1} \sin \left(\omega_{1} t-\pi / 2\right) \\
I_{2 a} & =I_{2} \sin \left(\omega_{2} t-\delta\right) \\
I_{2 b} & =I_{2} \sin \left(\omega_{2} t-\delta-\pi / 2\right)
\end{aligned}
$$

where $\delta$ is a phase delay between the primary coils and the secondary coils. And $I_{1}$ and $\omega_{1}$ are amplitude and angular frequency of the current of the primary coils, respectively. $I_{2}$ and $\omega_{2}$ are those of the secondary coils as well.

By substituting (10)-(13) to (1)-(8), the voltage equation is transformed as follows.

$$
\begin{aligned}
V_{1 a} & =R_{1} I_{1} \sin \left(\omega_{1} t\right)+\left(\omega_{1} L_{1}-\frac{1}{\omega_{1} C_{1}}\right) I_{1} \cos \left(\omega_{1} t\right) \\
& +\left(\omega_{2}+\omega\right) M_{0} I_{2} \cos \left(\omega_{2} t+\theta-\delta\right) \\
V_{1 b} & =-R_{1} I_{1} \cos \left(\omega_{1} t\right)+\left(\omega_{1} L_{1}-\frac{1}{\omega_{1} C_{1}}\right) I_{1} \sin \left(\omega_{1} t\right) \\
& +\left(\omega_{2}+\omega\right) M_{0} I_{2} \sin \left(\omega_{2} t+\theta-\delta\right) \\
V_{2 a} & =R_{2} I_{2} \sin \left(\omega_{2} t-\delta\right) \\
& +\left(\omega_{2} L_{2}-\frac{1}{\omega_{2} C_{2}}\right) I_{2} \cos \left(\omega_{2} t-\delta\right) \\
& +\left(\omega_{1}-\omega\right) M_{0} I_{1} \cos \left(\omega_{1} t-\theta\right) \\
V_{2 b} & =-R_{2} I_{2} \cos \left(\omega_{2} t-\delta\right) \\
& +\left(\omega_{2} L_{2}-\frac{1}{\omega_{2} C_{2}}\right) I_{2} \sin \left(\omega_{2} t-\delta\right) \\
& +\left(\omega_{1}-\omega\right) M_{0} I_{1} \sin \left(\omega_{1} t-\theta\right)
\end{aligned}
$$

In addition, the generated force (9) is also transformed into the form:

$$
F=\frac{2 \pi}{l_{x}} I_{1} I_{2} M_{0} \sin \left(\left(\omega_{1}-\omega_{2}\right) t-\theta+\delta\right) .
$$

Here, we can assume short circuits in the secondary side as same as the conventional induction machines. From (16) and 
(17), we have solutions satisfying $V_{2 a}=V_{2 b}=0$ as follows.

$$
\begin{aligned}
\delta & =-\tan ^{-1} \frac{R_{2}}{\omega_{2} L_{2}-\frac{1}{\omega_{2} C_{2}}} \\
I_{2} & =-\frac{\omega_{2} M_{0}}{\sqrt{R_{2}^{2}+\left(\omega_{2} L_{2}-\frac{1}{\omega_{2} C_{2}}\right)^{2}}} I_{1} \\
\omega_{2} t & =\omega_{1} t-\theta \\
\omega_{2} & =\omega_{1}-\omega .
\end{aligned}
$$

The phase delay $\delta$, the amplitude $I_{2}$ and angular frequency $\omega_{2}$ of the secondary current are determined uniquely.

By substituting these solutions (19)-(22) to (14) and (15), the voltage equations in the primary circuit are simply expressed as follows.

$$
\begin{aligned}
V_{1 a} & =I_{1} \sqrt{R_{1}^{2}+\left(\omega_{1} L_{1}-\frac{1}{\omega_{1} C_{1}}\right)^{2}} \cos \left(\omega_{1} t+\gamma\right) \\
& +\omega_{1} M_{0} I_{2} \cos \left(\omega_{1} t-\delta\right) \\
V_{1 b} & =I_{1} \sqrt{R_{1}^{2}+\left(\omega_{1} L_{1}-\frac{1}{\omega_{1} C_{1}}\right)^{2}} \sin \left(\omega_{1} t+\gamma\right) \\
& +\omega_{1} M_{0} I_{2} \sin \left(\omega_{1} t-\delta\right) \\
\gamma & =-\tan ^{-1} \frac{R_{1}}{\omega_{1} L_{1}-\frac{1}{\omega_{1} C_{1}}} .
\end{aligned}
$$

The generated force is also simplified as follows by substituting the solutions (19)-(22) to (18).

$$
F=\frac{2 \pi}{l_{x}} \frac{\left(\omega_{1}-\omega\right) M_{0}^{2} R_{2}}{R_{2}^{2}+\left(\omega_{2} L_{2}-\frac{1}{C_{2} \omega_{2}}\right)^{2}} I_{1}^{2} .
$$

From this result, it turns out that magnitude of the force can be controlled by the amplitude of the primary current $I_{1}$ and direction of the force can be controlled by the sign of the angular frequency of the primary currents $\omega_{1}$.

Further calculation yields more simplified expressions as follows.

$$
\begin{aligned}
& V_{1 a}=I_{1} A \cos \left(\omega_{1} t+\beta\right) \\
& V_{1 b}=I_{1} A \sin \left(\omega_{1} t+\beta\right)
\end{aligned}
$$

where

$$
\begin{aligned}
& A= \\
& \sqrt{R_{1}^{2}+X_{1}^{2}+\frac{\omega_{1} \omega_{2} M_{0}^{2}\left(\omega_{1} \omega_{2} M_{0}^{2}+2 R_{1} R_{2}-2 X_{1} X_{2}\right)}{R_{2}^{2}+X_{2}^{2}}} \\
& \beta=\tan ^{-1} \frac{\omega_{1} \omega_{2} M_{0}^{2} R_{2}+R_{1}\left(R_{2}^{2}+X_{2}^{2}\right)}{\omega_{1} \omega_{2} M_{0}^{2} X_{2}-X_{1}\left(R_{2}^{2}+X_{2}^{2}\right)} \\
& X_{1}=\omega_{1} L_{1}-\frac{1}{\omega_{1} C_{1}} \\
& X_{2}=\omega_{2} L_{2}-\frac{1}{\omega_{2} C_{2}} .
\end{aligned}
$$

Regarding the generated force, we have

$$
F=\frac{2 \pi}{l_{x}} \frac{\left(\omega_{1}-\omega\right) M_{0}^{2} R_{2}}{D} V_{1}^{2}
$$

where

$$
\begin{aligned}
& D=\left(R_{1}^{2}+X_{1}^{2}\right)\left(R_{2}^{2}+X_{2}^{2}\right) \\
& +\omega_{1} \omega_{2} M_{0}^{2}\left(\omega_{1} \omega_{2} M_{0}^{2}+2 R_{1} R_{2}-2 X_{1} X_{2}\right)
\end{aligned}
$$

and $V_{1}=\sqrt{V_{1 a}^{2}+V_{1 b}^{2}}$ is the amplitude of the voltage source in the primary circuits.

As same as typical induction machines, when extremely high frequency $\omega_{1}$ for the voltage source in the primary side is given, the slip frequency $\omega_{2}$ is also extremely high according to (22) because typical range of the operating frequency of the mover is less than $10 \mathrm{kHz}$. In a case of conventional induction machines, the secondary current hardly flows in such high frequency region because reactance of the coils in the secondary side is quite high. In contrast, the large secondary current flows in our proposed electric machine when the slip frequency $\omega_{2}$ is close to the resonant frequency. This point is quite different from the conventional induction machines. Therefore, the coils and the capacitors of the proposed machine should be designed so that the resonant frequency is quite higher than the operating frequency of the mover and the Q factor covers variation of the slip frequency due to the change of the operating frequency of the mover.

\section{RESONANT FREQUency}

In this section, the resonant frequencies are derived considering the effect of the speed of the secondary side mover. For simplicity, we assume that $R_{1}=R_{2}=0$ in this section. The condition that the impedance of the whole circuit becomes zero can be derived from the condition $A=0$ using (29) in this case, which is as follows.

$$
\omega_{1} \omega_{2} M_{0}^{2}-\left(\omega_{1} L_{1}-\frac{1}{\omega_{1} C_{1}}\right)\left(\omega_{2} L_{2}-\frac{1}{\omega_{2} C_{2}}\right)=0
$$

This condition is equivalent to $D=0$ in (34) when $R_{1}=$ $R_{2}=0$. We can see that this condition is independent from the electric angle $\theta$ of the mover. It means that the resonant frequency does not affected by the displacement of the mover. However, the resonant frequency depends on the slip frequency $\omega_{2}\left(=\omega_{1}-\omega\right)$ which also depends on $\omega$, the frequency of the mover.

If we assume that the inductances and the capacitance of the primary coils and the secondary coils are same, we can solve (35) with respect to $\omega_{1}$ under the conditions of $L_{1}=$ $L_{2}=L, C_{1}=C_{2}=C$, and $\omega_{2}=\omega_{1}-\omega$. As the results, we obtain four solutions in complicated forms. The Taylor series expansion of the solution with respect to $\omega$ around $\omega=0$ up to 1 st order is obtained as follows.

$$
\begin{gathered}
\omega_{1 \text { res }}=\left\{-\frac{1}{\sqrt{\left(L-M_{0}\right) C}}+\frac{\omega}{2},-\frac{1}{\sqrt{\left(L+M_{0}\right) C}}+\frac{\omega}{2},\right. \\
\left.\frac{1}{\sqrt{\left(L+M_{0}\right) C}}+\frac{\omega}{2}, \frac{1}{\sqrt{\left(L-M_{0}\right) C}}+\frac{\omega}{2}\right\} .
\end{gathered}
$$

They represent good approximations of the resonant frequencies. From these solutions, we confirm that there are four resonant frequencies in the proposed electric machine; two are in negative frequency domain and the other two are in positive frequency domain. All resonant frequencies are shifted by a half of the frequency of the mover. 


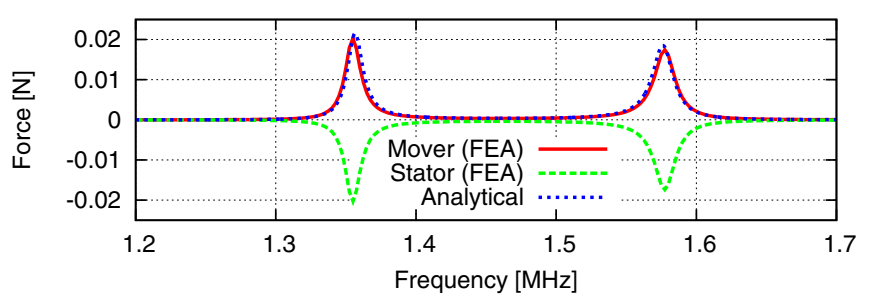

(a) Gap length: $l_{y}=10[\mathrm{~mm}]$

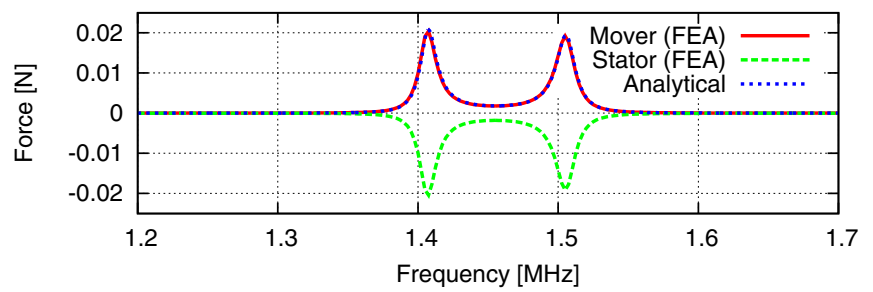

(b) Gap length: $l_{y}=15[\mathrm{~mm}]$

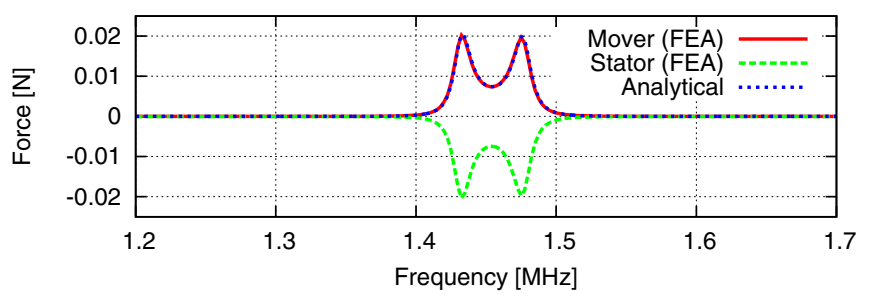

(c) Gap length: $l_{y}=20[\mathrm{~mm}]$

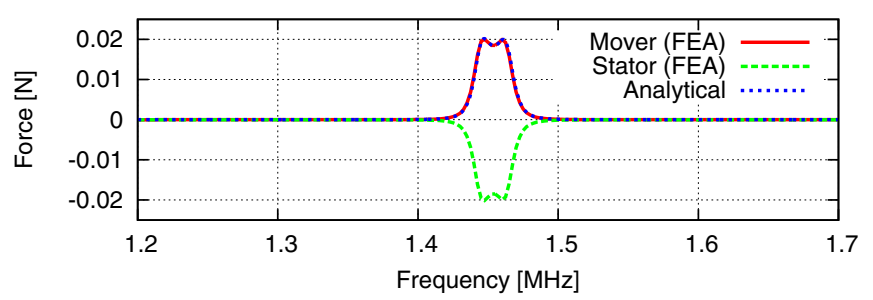

(d) Gap length: $l_{y}=25[\mathrm{~mm}]$

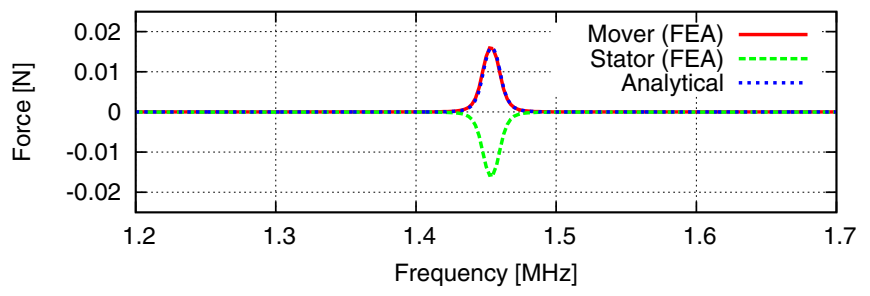

(e) Gap length: $l_{y}=30[\mathrm{~mm}]$

Fig. 3. FEA simulation results of force in tangent direction.

\section{FEA Simulations}

In this section, we confirm the proposed model by Finite Element Analysis (FEA). Parameters used in these simulations are shown in Table I. Fig. 3-Fig. 5 show numerical results calculated by the proposed model and simulation results of frequency responses calculated by using a FEA simulation software JMAG-Designer 14.0. Especially Fig. 3 shows numerical results given by (33) and simulation results of generated force along with the moving direction of the mover in various gap length. A product of this force and the speed of the mover corresponds to the mechanical power of the machine.

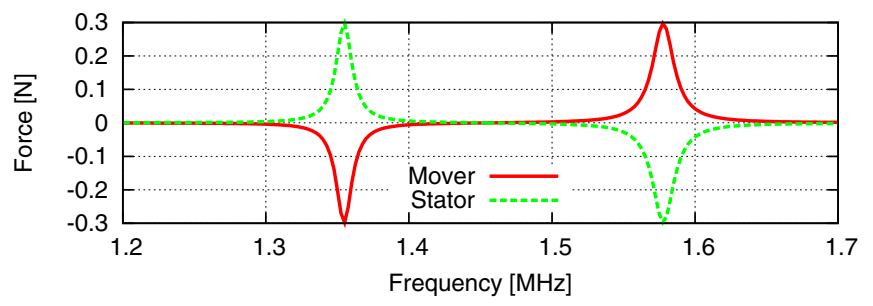

(a) Gap length: $l_{y}=10[\mathrm{~mm}]$

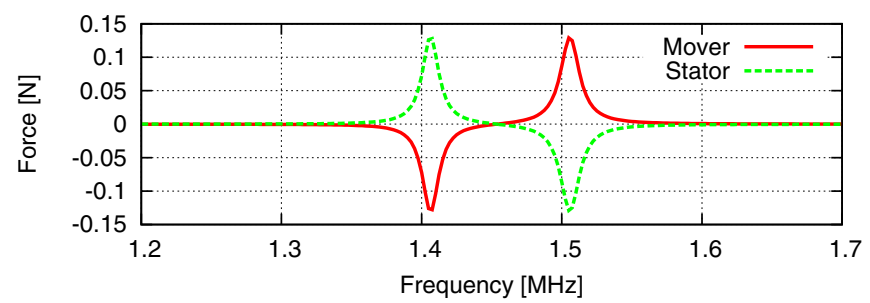

(b) Gap length: $l_{y}=15[\mathrm{~mm}]$

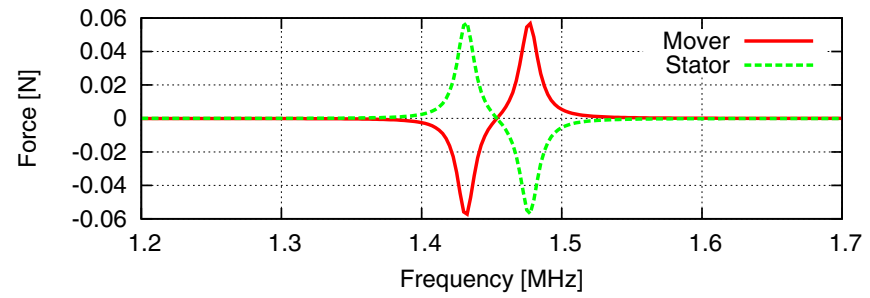

(c) Gap length: $l_{y}=20[\mathrm{~mm}]$

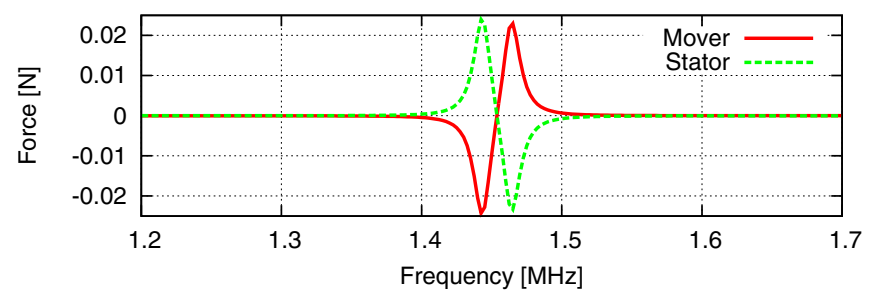

(d) Gap length: $l_{y}=25[\mathrm{~mm}]$

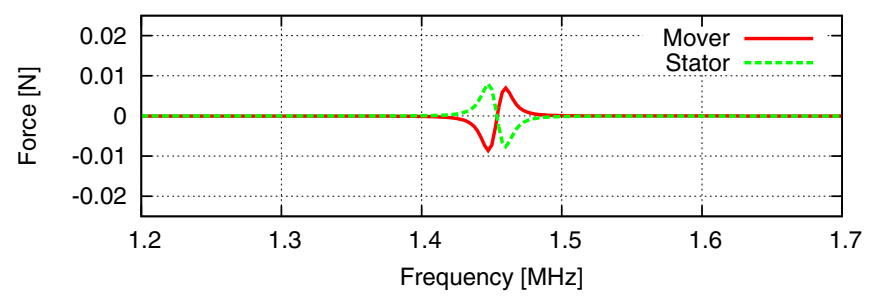

(e) Gap length: $l_{y}=30[\mathrm{~mm}]$

Fig. 4. FEA simulation results of force in normal direction.

We can confirm that the simulation results precisely agree with our proposed model (33). We can also observe two peak of the force at resonant frequencies. As gap length between the primary coils and the secondary coils increases, difference between two resonant frequencies becomes small. But magnitude of the force is almost constant until $l_{y} \leq 25$ $[\mathrm{mm}]$ and starts to decrease when $l_{y}>25[\mathrm{~mm}]$. This tendency will be strongly related to the scattering parameters. Fig. 4 shows simulation results of generated force perpendicular to the moving direction of the mover in various gap length. Repulsive force is generated at the lower resonant frequency 


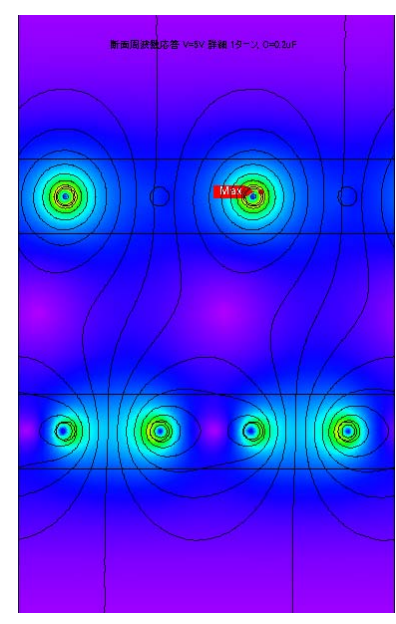

(a) At 0-degree phase angle.

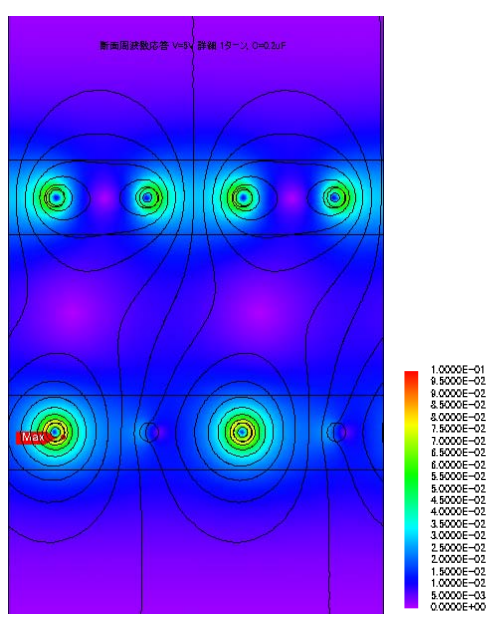

(b) At 45-degree phase angle.

Fig. 5. FEA simulation results of flux density under the condition of $l_{y}=$ $25[\mathrm{~mm}], \omega_{1}=1.45[\mathrm{MHz}]$.

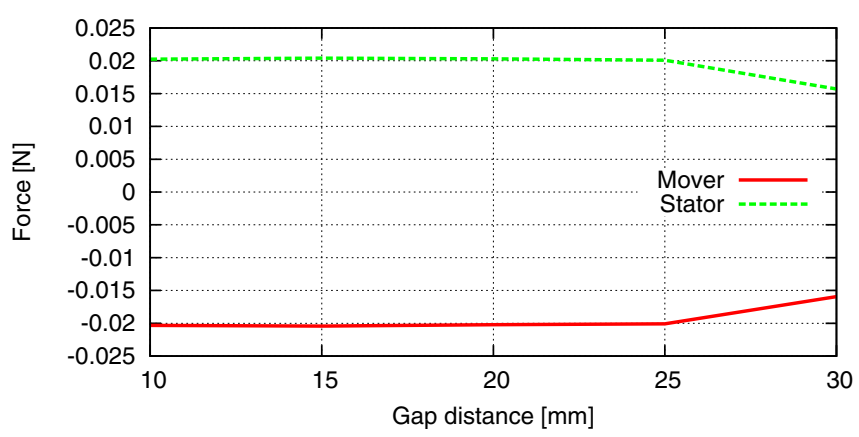

(a) Force in tangent direction.

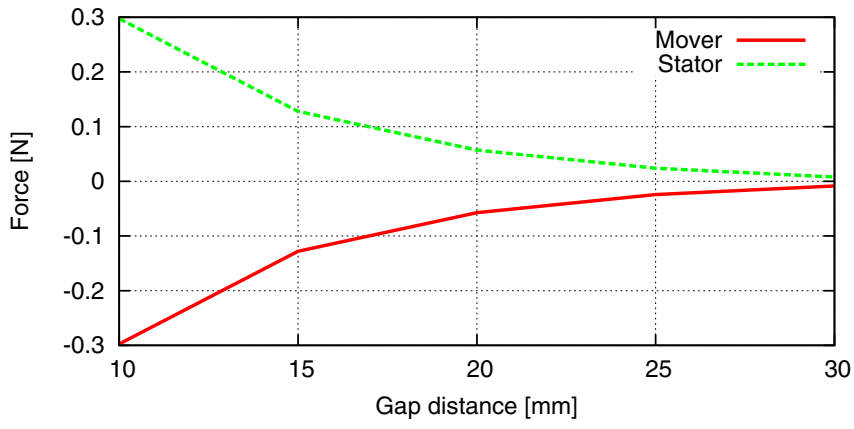

(b) Force in normal direction.

Fig. 6. Gap length dependency of force (FEA simulation).

while attractive force is generated at the higher resonant frequency. Fig. 5 shows contour plots of the flux density under the condition of the gap length $l_{y}=25[\mathrm{~mm}]$ and the frequency of the voltage source $\omega_{1}=1.45[\mathrm{MHz}]$.

Fig. 6 shows a gap length dependency of generated forces in the tangent direction (a) and in the normal direction (b). We can see that the normal force decreases as the gap length increases.

TABLE I. PARAMETERS OF THE MACHINE

\begin{tabular}{lll}
\hline Parameters & Values & Units \\
\hline \hline Pitch length of coils & $l_{x}=40$ & {$[\mathrm{~mm}]$} \\
Gap length & $10 \leq l_{y} \leq 30$ & {$[\mathrm{~mm}]$} \\
Depth of stator and mover & $l_{z}=50$ & {$[\mathrm{~mm}]$} \\
Amplitude of voltage source & $V_{1}=5$ & {$[\mathrm{~V}]$} \\
Resistance & $R_{1}=R_{2}=5.3$ & {$[\mathrm{~m} \Omega]$} \\
Capacitance & $C_{1}=C_{2}=0.22$ & {$[\mu \mathrm{F}]$} \\
The number of turn of coils & $N_{1}=N_{2}=1$ & \\
\hline
\end{tabular}

\section{CONCLUSION}

In this paper, we proposed an electro-mechanical energy conversion system using the resonant inductive coupling and examined the basic mathematical model of the system. This model unifies the wireless power transmission system and the electro-mechanical energy conversion system using the resonant inductive coupling. The proposed system is similar to the induction machine but it is driven at very high resonant frequency and the slip ratio is also quite high (near 100\%). The simulation results of the generated force by the FEA accurately agreed with the numerical results by the proposed model.

In the future, more detailed verification of the proposed model by simulations and experiments will be conducted. Improvement of the generated force is also another important subject. The proposed model will be applicable for various industrial applications such as cost-effective, light-weight largescale generators since the machine does not require precise gap design.

\section{APPENDIX}

The self-inductance and the mutual inductance are derived as follows. Consider a single straight wire, the number of turns of which is $n$, carrying current $I$ at the origin $O$ shown in Fig. 7. It generates magnetic field according to the Ampere's law. The normal component of the magnetic field is described as

$$
H_{0}=\frac{x}{2 \pi\left(x^{2}+y^{2}\right)} n I .
$$

The magnetic field at the position $(x, y)$ generated by the infinite number of wires located at the one-dimensional lattice as shown in Fig. 7 is calculated as follows.

$$
\begin{aligned}
H(x, y) & =\sum_{k=-\infty}^{\infty}\left(H_{0}\left(x-l_{x} k, y\right)-H_{0}\left(x-\frac{l_{x}}{2}(2 k-1), y\right)\right) \\
& =-\frac{2 \cosh \frac{2 \pi y}{l_{x}} \sinh \frac{2 \pi x}{l_{x}}}{l_{x}\left(\cos \frac{4 \pi x}{l_{x}}-\cosh \frac{4 \pi y}{l_{x}}\right)} n I
\end{aligned}
$$

Therefore, the self-flux linkage is computed as

$$
\begin{aligned}
\Phi_{L} & =\mu_{0} l_{z} n \int_{a}^{l_{x} / 2-a} H(x, 0) d x \\
& =-\frac{\mu_{0} l_{z} n^{2} I}{\pi} \log \tan \frac{\pi a}{l_{x}}
\end{aligned}
$$

where $\mu_{0}$ is the permeability of free space, $a$ is the radius of area of the wires, and $l_{z}$ is the depth of the wires, respectively. Hence, the self-inductance is given by

$$
L=-\frac{\mu_{0} l_{z} n^{2}}{\pi} \log \tan \frac{\pi a}{l_{x}} .
$$




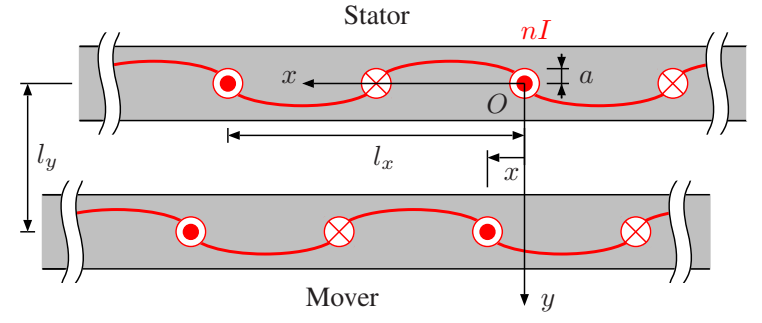

Fig. 7. A model for calculating self-inductance and mutual inductance.

The mutual inductance is also derived from the magnetic field (38). The interlinkage flux on the coil whose position is $\left(x, l_{y}\right)$ is calculated as follows.

$$
\begin{aligned}
\Phi_{M} & =\mu_{0} l_{z} n \int_{x}^{x+l_{x} / 2} H\left(x, l_{y}\right) d x \\
& =\frac{\mu_{0} l_{z} n^{2} I}{2 \pi} \log \frac{\cosh \frac{2 \pi l_{y}}{l_{x}}+\cos \frac{2 \pi x}{l_{x}}}{\cosh \frac{2 \pi l_{y}}{l_{x}}-\cos \frac{2 \pi x}{l_{x}}} .
\end{aligned}
$$

Therefore the mutual inductance between two coils is given by

$$
M=\frac{\mu_{0} l_{z} n^{2}}{2 \pi} \log \frac{\cosh \frac{2 \pi l_{y}}{l_{x}}+\cos \frac{2 \pi x}{l_{x}}}{\cosh \frac{2 \pi l_{y}}{l_{x}}-\cos \frac{2 \pi x}{l_{x}}} .
$$

This function can be approximated by the cosine function. Its fundamental component is given by

$$
\begin{aligned}
M_{0} & =\frac{2}{l_{x}} \int_{0}^{l_{x}} M \cos \frac{2 \pi x}{l_{x}} d x \\
& =\frac{2 \mu_{0} l_{z} n^{2}}{\pi} \exp \left(-\frac{2 \pi l_{y}}{l_{x}}\right) .
\end{aligned}
$$

\section{REFERENCES}

[1] O. H. Stielau and G. A. Covic, "Design of Loosely Coupled Inductive Power Transfer Systems," proc. Int. Conf. on Power System Technology, pp. 85-90, 2000.

[2] G. Vandevoorde and R. Puers, "Wireless Energy Transfer for Standalone Systems: A Comparison Between Low and High Power Applicability," Sensors and Actuators A, vol. 92, no. 1, pp. 305-311, 2001.

[3] Andre Kurs, Aristeidis Karalis, Robert Moffatt, J. D. Joannopoulos, Peter Fisher, and Marin Soljacic, "Wireless Power Transfer via Strongly Coupled Magnetic Resonances," Science, vol. 317, pp. 83-85, 2007.

[4] Reid R. Harrison, "Designing Efficient Inductive Power Links for Implantable Devices," Int. Symp. on Circuits and Systems, pp. 2080 2083, 2007.

[5] Benjamin L. Cannon, James F. Hoburg, Daniel D. Stancil, and Seth Copen Goldstein, "Magnetic Resonant Coupling As a Potential Means for Wireless Power Transfer to Multiple Small Receivers," IEEE Trans. on Power Electronics, vol. 24, no. 7, pp. 1819-1825, 2009.
[6] Zhen Ning Low, Raul Andres Chinga, Ryan Tseng, and Jenshan Lin, "Design and Test of a High-Power High-Efficiency Loosely Coupled Planar Wireless Power Transfer System," IEEE Trans. on Industrial Electronics, vol. 56, no. 5, pp. 1801-1812, 2009.

[7] Takehiro Imura, Hiroyuki Okabe, Toshiyuki Uchida, and Yoichi Hori, "Study on Open and Short End Helical Antennas with Capacitor in Series of Wireless Power Transfer using Magnetic Resonant Couplings," proc. IEEE Industrial Electronics Society Annual Conference, pp. 38483853, 2009.

[8] Takehiro Imura, Hiroyuki Okabe, and Yoichi Hori, "Basic Experimental Study on Helical Antennas of Wireless Power Transfer for Electric Vehicles by using Magnetic Resonant Couplings," proc. Vehicle Power and Propulsion Conference, pp. 936-940, 2009.

[9] Chih-Jung Chen, Tah-Hsiung Chu, Chih-Lung Lin, and Zeui-Chown Jou, "A Study of Loosely Coupled Coils for Wireless Power Transfer," IEEE Trans. on Circuits and Systems II: Express Briefs, vol. 57, no. 7, pp. 536-540, 2010.

[10] Takehiro Imura and Yoichi Hori, "Maximizing Air Gap and Efficiency of Magnetic Resonant Coupling for Wireless Power Transfer Using Equivalent Circuit and Neumann Formula," IEEE Trans. on Industrial Electronics, vol. 58, no. 10, pp. 4746-4752, 2011.

[11] Seung-Hwan Lee and Robert D. Lorenz, "Development and Validation of Model for 95\%-Efficiency 220-W Wireless Power Transfer Over a 30-cm Air Gap," Trans. on Industry Applications, vol. 47, no. 6, pp. 2495-2504, 2011.

[12] Alanson P. Sample, David A. Meyer, and Joshua R. Smith, "Analysis, Experimental Results, and Range Adaptation of Magnetically Coupled Resonators for Wireless Power Transfer," IEEE Trans. on Industrial Electronics, vol. 58, no. 2, pp. 544-554, 2011.

[13] Atsuo Kawamura and Tae-Woong Kim, "Proposed Equivalent Circuit and Parameter Identification Method for Electro-Magnetic Resonance Based Wireless Power Transfer," Journal of Electrical Engineering and Technology, vol. 8, no.4, pp. 799-807, 2013.

[14] Mohammad Bani Shamseh, Atsuo Kawamura, Itsuo Yuzurihara, and Atsushi Takayanagi, "A Wireless Power Transfer System Optimized for High Efficiency and High Power Applications," proc. IEEJ Int. Power Electronics Conference, pp. 2794-2801, 2014.

[15] Atsuo Kawamura, Kazuaki Ishioka, and Junji Hirai, "Wireless Transmission of Power and Information Through One High-frequency Resonant AC Link Inverter for Robot Manipulator Applications," IEEE Trans. on Industry Applications, vol. 32, no. 3, pp. 503-508, 1996.

[16] Junji Hirai, Tae-Woong Kim, and Atsuo Kawamura, "Practical Study on Wireless Transmission of Power and Information for Autonomous Decentralized Manufacturing System," IEEE Trans. on Industrial Electronics, vol. 46, no. 2, pp. 349-359, 1999.

[17] Junji Hirai, Tae-Woong Kim, and Atsuo Kawamura, "Wireless Transmission of Power and Information and Information for Cableless Linear Motor Drive," IEEE Trans.on Power Electronics, vol. 15, no. 1, pp. 2127, 2000

[18] Hideaki Abe, Hiroshi Sakamoto, and Koosuke Harada, "A Noncontact Charger Using a Resonant Converter with Parallel Capacitor of the Secondary Coil," IEEE Trans. on Industry Applications, vol. 36, no. 2, pp. 444-451, 2000.

[19] Junji Hirai, Tae-Woong Kim, and Atsuo Kawamura, "Study on Intelligent Battery Charging Using Inductive Transmission of Power and Information," IEEE Trans. on Power Electronics, vol. 15, no. 2, pp. 335-345, 2002. 